\title{
АЛГОРИТМ ВЫБОРА МЫШЦ МИШЕНЕЙ ДЛЯ ИНЪЕКЦИЙ ПРЕПАРАТАМИ БОТУЛИНИЧЕСКОГО ТОКСИНА ПАЦИЕНТАМ С ДЕТСКИМ ЦЕРЕБРАЛЬНЫМ ПАРАЛИЧОМ
}

\author{
${ }^{1}$ Носко А.С., ${ }^{1}$ Зыков В.П. \\ ${ }^{1}$ Кафедра неврологии детского возраста, РМАПО. Москва, РФ
}

\section{Резюме}

Ботулинический токсин типа А (БТА) как агент, воздействующий на локальную мышечную спастичность, в составе комплексной реабилитации пациентов с детским церебральным параличом (ДЦП) применяется лишь в течение последних двадцати лет. Данная статья основана на Международных консенсусах 2006, 2009 и 2010 гг. по применению ботулинотерапии в неврологической практике. В статье отражен алгоритм формирования целей ботулинотерапии, необходимые системы оценки и методики для выбора мышц-мишеней в соответствии с международными рекомендациями. А также было уделено внимание результатам рандомизированных исследований эффективности терапии спастичности БТА, возможным проблемам в определении показаний к лечению и перспективам метода.

Ключевые слова: ботулинический токсин, детский церебральный паралич, ДЦП.

Препараты ботулинического токсина

типа А (БТА) широко используются для коррекции локальной спастичности скелетных мышц при различных неврологических заболеваниях. Основным показанием к проведению инъекций БТА в педиатрической практике является детский церебральный паралич (ДЦП) - заболевание, в большинстве случаев сопровождающееся повышением мышечного тонуса и спастичностью. Изменение тонуса и мышечной силы, сохранение патологических рефлексов и синкинезий при ДЦП ведут к формированию патологической позы и нарушению двигательного стереотипа, что препятствует своевременному моторному развитию и социальной адаптации пациентов.

За последнее время произошло изменение взглядов на ботулинотерапию. Это отразились в изменении концепции «снижение мышечного тонуса при помощи БТА» на концепцию «улучшение общей моторной активности при помощи БТА». На основании этой позиции алгоритм выбора мышц мишеней для проведения инъекций препаратов ботулинического токсина представляет собой логичный переход от выявления особенностей моторного развития и характера двигательных нарушений у конкретного пациента на определенном этапе развития к решению данных проблем путём снижения спастичности в определяющих патологический паттерн мышцах.

Таким образом, ключевым моментом для выбора целевых мышц для инъекции БТА стало объективное определение уровня моторного развития ребёнка с ДЦП и ближайших целей реабилитации. Для этого применяют стандартизованные шкалы, использование которых необходимо при осмотре пациента на каждом этапе ботулинотерапии. Данный алгоритм и достоверные методики оценки детально описаны в Европейских консенсусах по применению ботулинотерапии для коррекции спастичности у детей с детским церебральным параличом 2006, 2009 и 2010 гг. (1-4).

Наиболее целесообразным считается проведение осмотров перед введением БТА, через 3-4 недели после - на высоте максимального эффекта и через 16-18 недель , что позволяет оценить уменьшение эффекта ботулинотерапии и возобновление спастичности, а так же решить вопрос о целесообразности и возможных сроках повторного введения БТА.

Каждый осмотр состоит из нескольких обязательных этапов.

\section{1.Определение уровня глобальной активности.}

Оценка уровня глобальной двигательной активности ребенка в привычных средовых условиях по 5-ти уровневой шкале GMFCS. Эта система является эквивалентом степени тяжести 
ДЦП (5-6).

Уровень I - ходьба без ограничений;

Уровень II - ходьба с ограничениями;

Уровень III - ходьба с использованием ручных приспособлений для передвижения;

Уровень IV - самостоятельное передвижение ограничено, могут использоваться моторизированные средства передвижения;

Уровень V - перевозка в ручном инвалидном кресле.

Цели бутолинотерапии отличаются в зависимости от уровня двигательной активности согласно системе GMFCS:

GMFCS I-III - улучшение походки и функции;

GMFCS III-V - коррекция позы;

GMFCS V - уменьшение выраженности болевого синдрома и/или дискомфорта, а так же облегчение ухода за пациентом.

В процессе прединъекционного осмотра родителей пациента необходимо информировать о глобальных целях терапии БТА, поскольку ориентирование на реально достижимый результат является залогом продуктивного взаимодействия и позитивного восприятия, достигнутого в результате лечения эффекта.

Так же в осмотр обязательно входит видеорегистрация движения (желательно провести компьютерный $3 \mathrm{D}$ видеоанализ, но при его отсутствии - видеосьемка на камеру). Это позволяет продумать схему инъекций согласно имеющемуся патологическому двигательному стереотипу, при необходимости скорректировать задачи и схему инъекций без участи ребенка, а так же зафиксировать результат лечения на каждом из постинъекционных этапов.

\section{2. Детальная оценка функций с} использованием объективизирующих шкал:

Составители консенсусов по ботулинотерапии рекомендуют шкалу GMFM66 (или 88 разработанную специально для ДЦП) как основной инструмент объективной и цифровой оценки степени освоения основных навыков: поворотов, удержания позы “сидя", “стоя”, ходьбы и бега у детей с ДЦП. Эта шкала является золотым стандартом для определения цели реабилитации пациента с ДЦП и позволяет объективно оценить динамику двигательного развития на фоне проводимой реабилитации.

Так же возможно применение шкал для детальной оценки функции верхней конечности (QUEST, AHA, BFBF) (7-10), для верификации степени социально-бытовой адаптации (WeeFIM, COMP) и т.д.

3. Определение конкретной схемы инъекций ботулинического токсина.

В консенсусе 2010 года по применению БТА в коррекции спастичности нижних конечностей у детей с ДЦП приведены результаты $29 \quad$ гетерогенных рандомизированных плацебо-контролируемых исследований по определению дозировки БТА при коррекции спастического эквинуса, профилактике дисплазии бедер, по проведению инъекционных сессий в различные группы мышц для улучшения походки и функционирования.

Суммируя полученные результаты, были выработаны следующие рекомендации:

- БТА является эффективным для коррекции спастического эквинуса с целью улучшения навыка ходьбы (уровень доказательств А)

- БТА введение в аддукторы бедер возможно эффективно при некоторых задачах, направленных на коррекцию ходьбы (уровень доказательств В)

- БТА инъекции в аддукторы бедер не повышает общий уровень двигательной активности (уровень доказательств А).

- БТА введение в аддукторы (и hamstringгруппу) может предотвратить дисплазию бедер, но не влияет на продолжительный прогноз (уровень доказательств А).

Представленные показания к проведению терапии БТА предполагают применение корректных методик выбора мышц мишеней:

- Исследование неврологического статуса по классической схеме.

- Оценку степени повышения тонуса м-ц по модифицированной шкале Тардье (11-13).

- Функциональную гониометрию $(14,15,17)$.

Целесообразным считается применение модифицированной шкалы Тардье (МШТ), в которой сохранена наиболее важная черта шкалы Тардье: определение угла резкой остановки движения в суставе («catch» (катч)- в англ. литературе). МШТ была признана 
экспертами как объективная, достоверная и чувствительная упрощенная система для оценки степени выраженности мышечной спастичности $(4,13)$.

Движение в суставе производится с разной скоростью: V1 - максимально медленно (минимизация рефлекса на растяжение); V2 Скорость падения сегмента конечности под воздействием гравитации; V3 - максимально быстро.

Объем движения в суставе определяется следующим образом:

R2 характеризует длину растяжения мышцы в спокойном состоянии (объем пассивного движения - V1) и R1 характеризует объем движения, на котором возникает резкое ограничение движения (так называемый «саtch» - англ.), обусловленное повышенным рефлексом на растяжение (V2 или V3). Поскольку V1 использую для оценки объема пассивного движения (ОПД), только V2 и V3 дает представление о спастичности. Важна именно разница между R1 и R2.

- Большая разница между R1 и R2 говорит о динамичности контрактуры и значительном объеме возможной положительной динамики на фоне коррекции.

- Незначительное различие между R1 и R2 связано с выраженностью фиксированной контрактуры.

Так же важной составляющей осмотра является функциональная гониометрия (ФГ), которая является логичным продолжением МШT. (1-4).

ФГ дает представление о вовлечении мышечных групп в формирование патологической позы и двигательного стереотипа, а так же о степени выраженности их истиной спастичности.

Выраженность истиной спастичности (без включения дистонического компонента) определяют путем совершения пассивного движения достаточно резким рывком, так называемый “catch" («замок», «стопор» - англ.), разнице между максимальным объемом пассивного движения и уровнем, на котором движение останавливается полностью при пробе на спастичность, и определяют как объем ограничения, обусловленный гипертонусом групп мышц-антагонистов. Разграничение спастики и дистонии крайне важно для выбора схемы инъекции БТА и позволяет в числовом выражении контролировать эффект от лечения. Система гониометрии детализирует мышцымишени согласно реабилитационным задачам, которые были поставлены на этапе определения глобального уровня двигательной активности, видео-регистрации и прицельной оценки движений с использованием шкал.

Итак, представленный алгоритм позволяет верифицировать влияние БТА на истинную спастичность, объем функционирования, что значительно объективизирует результат лечения. Это крайне важно на современном этапе развития ботулинотерапии, когда в педиатрической практике доказательства группы А получены только для коррекции гипертонуса мышц нижних конечностей при помощи БТА, на территории РФ - коррекции патологического эквинуса. Иные же показания к применению ботулинотерапии у детей (спастичность верхней конечности, шеи и т.д.) по-прежнему остаются в категории В и U, большей частью за счет отсутствия стандартизированной системы формирования реабилитационной задачи, оценки результатов лечения и неоднородности исследуемых групп (3).

\section{СПИСОК ЛИТЕРАТУРЫ}

1. F. Heinen, K. Desloovere, A. S. Schroeder et. al. The updated European Consensus 2009 on the use of Botulinum toxin for children with cerebral palsy. European Journal of Paediatric Neurology 2010; 14: 45-66.

2. F. Heinen, K. Desloovere, A. S. Schroeder et. al. Europian consensus table 2006 on botulinum toxin for children with cerebral palsy. European Journal of Pediatric Neurology 2006; 10: 215-225.

3. A. Esquenazi, I. Novak, G. Sheean, B.J. Singerand A.B.Ward. International consensus for the use of botulinum toxin treatment in adalts and children with neurological impairments introduction. European Journal of Neurology 2010; 17 (Suppl. 2): 1-8.

4. S.C. Love, I. Novak, M. Kentish, K. Desloovere et al. Botulinum toxin assessment, 
intervention and after-care for lower limb spasticity in children with cerebral palsy: international

5. Palisano RJ, Rosenbaum $\mathrm{P}$, Walter $\mathrm{S}$, Russell D, Wood E, Galuppi B. Development and reliability of a system to classify gross motor function in children with cerebral palsy. Dev Med Child Neurol 1997; 45: 113-120.

6. Palisano RJ, Hanna SE, Rosenbaum PL, Russell DJ, Walter SD, Wood EP, et al. Validation of a model of gross motor function for children with cerebral palsy. Phys Ther 2000; 80(10):97485 .

7. Lowe K, Novak I, Cusick A. Lowdose/high-concentration localized Botulinum toxin A improves upper limb movement and function in children with hemiplegic cerebral palsy. Dev Med Child Neurol 2006;48(3):170-5.

8. Wallen M, O'Flaherty SJ, Waugh MC. Functional outcomes of intramuscular Botulinum toxin type a and occupational therapy in the upper limbs of children with cerebral palsy: a randomized controlled trial. Arch Phys Med Rehabil 2007; 88(1):1-10.

9. Krumlinde-Sundholm L, Holmefur M, Kottorp A, Eliasson AC. The Assisting Hand Assessment: current evidence of validity, reliability, and responsiveness to change. Dev Med Child Neurol 2007;49(4):259-64.

10. Beckung E, Hagberg G. Neuroimpairments, activity limitations, and participation restrictions in children with cerebral palsy. Dev Med Child Neurol 2002;44(5):309-16.

11. Damiano DL, Quinlivan JM, Owen BF, Payne P, Nelson KC, Abel MF. What does the Ashworth scale really measure and are instrumented measures more valid and precise? Dev Med Child Neurol 2002;44(2):112-8.

12. Scholtes VA, Becher JG, Beelen A, Lankhorst GJ. Clinical assessment of spasticity in children with cerebral palsy: a critical review of available instruments. Dev Med Child Neurol 2006;48(1):64-73.

13. Boyd RN, Graham HK. Objective measurement of clinical findings in the use of Botulinum toxin type A for the management of children with cerebral palsy. European Journal of Neurology 1999;6(Suppl. 4):23-35.

14. Allington NJ, Leroy N, Doneux C. Ankle joint range of motion measurements in spastic cerebral palsy children: intraobserver and interobserver reliability and reproducibility of goniometry and visual estimation. J Pediatr Orthop B 2002;11(3):236-9.

15. McDowell BC, Hewitt V, Nurse A, Weston $\mathrm{T}$, Baker R. The variability of goniometric measurements in ambulatory children with spastic cerebral palsy. Gait Posture 2000;12(2): 114-21.

16. Palisano RJ, Rosenbaum P, Walter S, Russell D, Wood E, Galuppi B. Development and reliability of a system to classify gross motor function in children with cerebral palsy. Dev Med Child Neurol 1997; 45: 113-120.

17. Fox J., Day R. A Physiotherapist's Guide to Clinical Measurement. Churchill Livingstone. 2009; 288 p.

\section{SUMMARY}

\section{ALGORITHM OF MUSCLES SELECTION FOR BOTULINUM TOXIN INJECTIONS AT PATIENTS WITH CEREBRAL PALSY}

Nosko A., Zykov V.

\section{Pediatrician Neurology Department, RMAPA. Moscow, $R F$}

Botulinum neurotoxin type-A (BoNT-A) has been used in association with other interventions in the management of spasticity in children with cerebral palsy (CP) for almost two decades. This article is based on review of International Consensus statements 2006, 2009 and 2010. In the article the algorithm of botulinum toxin therapy goals determination, the list of necessary assessment scales and protocols of injected muscles determination have been described. Attention has been paid to randomized clinical trials, possible complications and method perspectives.

Key words: botulinum toxin, cerebral palsy, CP.

\section{XÜLASə}

UŞAQ SEREBRAL İFLICLI PASIYYENTLORə BOTULIN TOKSINI PREPARATLARININ İNYEKSIYYASI ÜÇÜN HODӘF OZӘLOLӘRIN SEÇIMI ALQORITMI 
Nosko A.S., Zıkov V.P.

Uşaq yaşlarının nevrologiyası kafedrası, Rusiya Diplomdansonrakı Tibb Akademiyası, Moskva,

Rusiya

A tip botulin toksini (BTA) uşaq serebral iflici (USI) olan pasiyentlərdə kompleks reabilitasiya tərkibində lokal əzələ spastikliyinə təsir göstərən agent kimi ancaq son 20 il ərzində istifadə edilir. Təqdim edilmiş məqalə nevroloji praktikada botulinoterapiyanın istifadəsinin 2006, 2009 və 2010-cu illərin Beynəlxalq konsensuslarına əsaslanır. Məqalədə beynəlxalq tövsiyələrə uyğun hədəf əzələlərin seçilməsi üçün botulinoterapiyanın məqsədlərinin formalaşdırılması alqoritmi, vacib qiymətləndirmə və metodika sistemləri əks olunmuşdur. Həm də, məqalədə əzələ spastikliyinin BTA ilə müalicəsinin effektivliyinin randomizəolunmuş tədqiqatlarının nəticələrinə, bu metodun perspektivlərinin və müalicəуə göstərişlərin təyininin mümkün problemlərinə diqqət ayrılmışdır.

Açar sözlər: botulin toksini, uşaq serebral iflici, USİ 